\title{
External Ear Polyp
}

National Cancer Institute

\section{Source}

National Cancer Institute. External Ear Polyp. NCI Thesaurus. Code C4366.

A benign polypoid growth in the external ear. 